\title{
Understanding Social Costs in Online Question Asking
}

\author{
Haiwei Ma \\ University of Minnesota \\ Minneapolis, MN, USA \\ maxxx979@umn.edu

\section{Hao Fei Cheng} \\ University of Minnesota \\ Minneapolis, MN, USA \\ cheng635@umn.edu
}

\author{
Bowen Yu \\ University of Minnesota \\ Minneapolis, MN, USA \\ bowen-yu@umn.edu \\ Haiyi Zhu \\ University of Minnesota \\ Minneapolis, MN, USA \\ zhux0449@umn.edu
}

\begin{abstract}
Various social media sites and online communities provide new channels for people in need to ask questions and seek help. However, individuals may still encounter psychological barriers that deter solicitation for assistance, which is more formally described as "social costs". For example, it can be the concerns of burdening others, the obligation of reciprocation, etc. To understand what could reduce social costs, we conducted a study in the context of Question-Answering (QA) and investigated the following three factors inspired by literature: anonymity (posting a question anonymously), recommendation (having the system handle the question routing), and ephemerality (allowing questions to be visible only for a short period). We built a QA platform to support these three features and conducted a randomized within-subject experiment to test their effects on social costs of posting questions. Results suggest the presence of anonymity, recommendation, and ephemerality reduces the social costs which provides design implications for future community building.

Permission to make digital or hard copies of part or all of this work for personal or classroom use is granted without fee provided that copies are not made or distributed for profit or commercial advantage and that copies bear this notice and the full citation on the first page. Copyrights for third-party components of this work must be honored. For all other uses, contact the owner/author(s)

CHI'19 Extended Abstracts, May 4-9, 2019, Glasgow, Scotland UK

(c) 2019 Copyright held by the owner/author(s).

ACM ISBN 978-1-4503-5971-9/19/05.

https://doi.org/10.1145/3290607.3313042
\end{abstract}


Social costs are psychological burdens of seeking help from others. It might contain the following perspectives:

- Incompetence: the cost of acknowledging incompetence

- Dependence: the cost of acknowledging dependence

- Indebtedness: the cost of feeling obligated to reciprocate

- Control: the cost of losing control over the problem

- Privacy: the cost of disclosing sensitive information

- Development: the cost of losing the opportunity to solve the problem alone

- Relationship: the cost of exploiting the relationship

- Self-image: the cost of harming self-image

- Effort: the cost of spending time and effort to ask for help

- Consideration: the cost of bothering others

- Self-confidence: the cost of conveying lack of self-confidence

- Unease: the cost of inducing uncomfortable feelings

\section{Sidebar 1: How we describe social} costs.

\section{CCS CONCEPTS}

- Human-centered computing $\rightarrow$ Contextual design.

\section{KEYWORDS}

Social Costs; Online Question Asking; Anonymity; Recommendation; Ephemerality; System Building

\section{ACM Reference Format:}

Haiwei Ma, Bowen Yu, Hao Fei Cheng, and Haiyi Zhu. 2019. Understanding Social Costs in Online Question Asking. In CHI Conference on Human Factors in Computing Systems Extended Abstracts (CHI'19 Extended Abstracts), May 4-9, 2019, Glasgow, Scotland UK. ACM, New York, NY, USA, 6 pages. https://doi.org/10.1145/3290607.3313042

\section{INTRODUCTION}

People sometimes are reluctant to ask questions or help to others. To name a few examples. People may hesitate to ask strangers for help to finish a task [11]. People with mental issues such as depression have difficulty in asking questions or help from health processionals [2]. Even with the development of various online communities, platforms, and technologies that makes connect with others much easier, unwillingness of asking questions or seeking help is still a barrier for many people. Brady et al. developed an iPhone application, VizWiz Social, that lets a blind person take a picture, ask a question they'd like to know about the picture, and then get an answer back from sighted users on VizWiz [4] However, they found that blind users hesitated to use VizWiz to solicit help because they considered asking questions as a burden. Frey and Seaman empirically investigated the help seeking behavior of user-end software engineers by conducting storytelling interviews and identified approaches, such as providing tools or environments, to provide support [6], however, the factor that can cause barriers for help seeking is still unknown.

Social scientists have identified a set of factors that may deter people in need from question asking and help seeking. For example, the concern of burdening others [4], the risk of exploiting friendship [10], the obligation to repay help givers [13], the concern of harming self-image [7], etc. We refer these factors as "social costs" associated with help seeking.

The goal of this paper is to investigate ways of reducing social costs in the context of online Question Answering (QA). We identify twelve perspectives of social costs from the literature, and propose three features that could reduce social costs associated with online QA: anonymity (posting a question anonymously instead of disclosing real identity), recommendation (having the system instead of users pick potential helpers), and ephemerality (allowing questions to be visible for only a short period instead of a relatively long period).

We build our own QA platform, Mobilyzr, to support these three features and run a randomized within-subject experiment to study social costs of posting questions in different conditions. We find 
For each perspective of social costs, we ask participates to rate: "To what extent do you agree with the following statements describing your experience of posting this question?" 1 = Strongly Disagree and 5 = Strongly Agree.

- Incompetence: It makes me feel inadequate or incompetent.

- Dependence: It threatens my ethic of independence or my inclination to do something on my own.

- Indebtedness: It makes me feel that I need to reciprocate to the answerers.

- Control: I feel that it will influence my own answer in a way I don't want.

- Privacy: I feel that it discloses important information that I don't want others to know.

- Development: I feel that it takes away my chance to do and learn something, or affects my learning process negatively in other ways.

- Relationship: It makes me feel that I am exploiting the relationship.

- Self-image: I feel that it will incur negative judgments from others.

- Effort: I feel that it takes more time and effort than solving the problem alone.

- Consideration: I feel that it will bother others in a way I don't want.

- Self-confidence: I feel that it conveys a lack of confidence on my part.

- Unease: It makes me feel uncomfortable.

Sidebar 2: Questions to measure 12 types of social costs. that anonymity reduces perceived costs of "repaying” answerers (indebtedness), recommendation reduces perceived costs of requesters' valuation of others' expending time, attention, and effort, and ephemerality reduces perceived costs of requesters' curated online persona (self-image) and privacy concerns. The results confirm that anonymity, recommendation and ephemerality can decrease social costs associated with online question asking, and provide implications for future community design.

\section{SOCIAL COSTS IN SOCIAL PSYCHOLOGY}

Social psychology has a long tradition of studying social costs, focused on the definition, measurement and causes.

Researchers identified various concerns people have when seeking assistance, such as its implication of incompetence and inferiority [5, 7], or a sign of dependence on others [8]. People might feel indebted and obligated to reciprocate a favor after receiving aid [13], and sometimes aid requests may involve sensitive information that the requester doesn't want to disclose [14]. This list can go longer, but yet there is no universal definition or taxonomy about social costs. Hence, we only focus on the types of social costs that have been identified and are relevant to the context of online QA. We formally described twelve perspectives of social costs by a thorough literature review (see Sidebar 1).

A common way to measure social costs by social psychologists is to conduct questionnaires. For instance, Anderson et al. designed a questionnaire that consists of 7 items to measure social costs of asking questions at work [1], and Lee et al. designed one that includes 3 items to measure incompetence and dependence [8]. We adapted from the literature and designed our own questionnaire to capture the 12 perspectives we identified (see Sidebar 2).

Social psychologists have contributed immense amounts of work to identify factors that influence question-asking behaviors, which can be summarized into four categories: individual, relational, task, and contextual factors.

Contextual factors refer to the context or the situation where question-asking occurs, which are easier for system designers to manipulate and operate to reduce social costs, especially in online settings. To list a few examples. Anonymity is a common strategy for people in online peer questionasking communities like Alcoholics Anonymous communities, where people don't want to disclose their identify [15]. Social recommendation is another way that facilitates people to reach out [12] With the rise of Snapchat, ephemerality has been considered as another social cost reducer [3].

All these three features, anonymity, recommendation, and ephemerality, have in some way played a role to facilitate people's communication. In this paper, we formally consider them as contextual factors and investigate their effects on reducing social costs. 


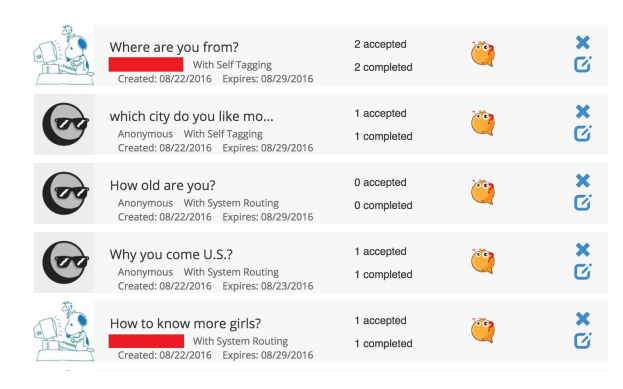

Figure 1: Description about experiment condition each question is assigned to.

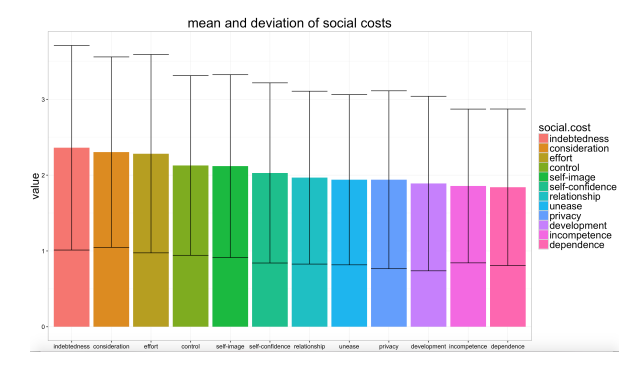

Figure 2: Means and deviations of 12 types of social costs.

\section{METHODS}

\section{Study Platform - Mobilyzr}

We built a Question Answering platform, Mobilyzr, on top of the Facebook social graph for a randomized within-subject experiment. Only participants who registered on the platform can post, browse, and answer questions of others. We operationalized these three features in the system as following: for anonymity, questions are either (1) distributed anonymously, or (2) attached with requesters' real names; for recommendation, questions are distributed to two other users (1) who were randomly selected by the system, or (2) who were tagged by the question requester; for ephemerality, questions are visible in the system for (1) 1 day (a shorter time period), or 7 days (a longer time period). Our system sends a notification email to users who are either tagged by the requester or recommended by the system to answer the question. A screenshot of Mobilyzr is depicted in Figure 1.

\section{Study Design and Procedure}

We adopted a within-subject design to mitigate the effects of individual differences with $8(2 \times 2 \times 2)$ conditions. All the questions asked by participants are randomly assigned to one of the eight conditions. Thus, participants could not tailor their questions based on assigned conditions.

We employed a snowball recruiting process to sample a network mixed with strangers and friends as a simulation of real-world networks. The recruitment started from researchers' own social networks, and then invited participants were free to introduce their friends to join our study. As a result, we recruited 31 participants (23 Male, 8 Female).

We started our study by explaining our system and study procedure to participants. After clarification, participants signed the consent forms voluntarily, and created their accounts on Mobilyzr using their Facebook accounts. Participants were given one month to post at least eight questions of any topic in their interest. We did not impose any requirement on what nor how to post to simulate a natural experiment. Each question was assigned to one of the eight conditions in a random order Once the question was posted, it would be shown on the page (Figure 1). The system logged all the activities of participants, including how long they responded a question and the content of the response. In total, we collected 244 questionnaire responses for our analysis.

One month later when the study was over, we conducted a 30-minute interview with participants asking about their experience and thoughts while using the platform. Participants were then debriefed and compensated.

\section{Study Analysis}

Dependent Variable. Social Costs. We have twelve variables - each to measure one perspective of social costs (see Sidebar 2), in a 5-point Likert scale, with 1 = Strongly Disagree and $5=$ Strongly Agree. 


\begin{tabular}{|c|c|c|c|c|}
\hline & \multicolumn{2}{|c|}{ Incompetence } & \multicolumn{2}{|c|}{ Dependence } \\
\hline & Coef. & $P$ & Coef. & $\mathrm{P}$ \\
\hline Intercept & 1.932 & *** & 1.903 & *** \\
\hline Anonymity & -0.104 & 0.250 & -0.052 & 0.599 \\
\hline Recommendation & -0.009 & 0.921 & 0.012 & 0.903 \\
\hline \multirow[t]{3}{*}{ Ephemerality } & -0.038 & 0.675 & -0.085 & 0.391 \\
\hline & \multicolumn{2}{|c|}{ Indebtedness } & \multicolumn{2}{|c|}{ Control } \\
\hline & Coef. & $\mathrm{P}$ & Coef. & $\mathrm{P}$ \\
\hline Intercept & 2.522 & $* * *$ & 2.202 & $* * *$ \\
\hline Anonymity & -0.227 & $0.022^{*}$ & 0.060 & 0.583 \\
\hline Recommendation & -0.096 & 0.332 & -0.026 & 0.812 \\
\hline \multirow[t]{3}{*}{ Ephemerality } & 0.003 & 0.973 & -0.184 & 0.095 \\
\hline & \multicolumn{2}{|c|}{\begin{tabular}{|l|} 
Privacy \\
\end{tabular}} & \multicolumn{2}{|c|}{ Development } \\
\hline & Coef. & $P$ & Coef. & $\mathrm{P}$ \\
\hline Intercept & 2.206 & $* * *$ & 1.928 & $* * *$ \\
\hline Anonymity & -0.057 & 0.622 & -0.004 & 0.972 \\
\hline Recommendation & -0.141 & 0.225 & -0.102 & 0.332 \\
\hline \multirow[t]{3}{*}{ Ephemerality } & -0.335 & $0.004^{* *}$ & 0.028 & 0.787 \\
\hline & \multicolumn{2}{|c|}{ Relationship } & \multicolumn{2}{|c|}{ Self-image } \\
\hline & Coef. & $\mathrm{P}$ & Coef. & $\mathrm{P}$ \\
\hline Intercept & 2.060 & $* * *$ & 2.418 & $* * *$ \\
\hline Anonymity & -0.162 & 0.101 & -0.206 & 0.062 \\
\hline Recommendation & -0.041 & 0.675 & -0.083 & 0.450 \\
\hline \multirow[t]{3}{*}{ Ephemerality } & 0.020 & 0.841 & -0.306 & $0.006^{* *}$ \\
\hline & \multicolumn{2}{|c|}{ Effort } & \multicolumn{2}{|c|}{ Consideration } \\
\hline & Coef. & $\mathrm{P}$ & Coef. & $\mathrm{P}$ \\
\hline Intercept & 2.501 & $* * *$ & 2.466 & $* * *$ \\
\hline Anonymity & -0.080 & 0.518 & 0.061 & 0.583 \\
\hline Recommendation & -0.202 & 0.101 & -0.269 & $0.016^{*}$ \\
\hline \multirow[t]{3}{*}{ Ephemerality } & -0.152 & 0.216 & -0.117 & 0.292 \\
\hline & \multicolumn{2}{|c|}{ Self-confidence } & \multicolumn{2}{|c|}{ Unease } \\
\hline & Coef. & $\mathrm{P}$ & Coef. & $\mathrm{P}$ \\
\hline Intercept & 2.175 & $* * *$ & 1.989 & $* * *$ \\
\hline Anonymity & 0.020 & 0.871 & -0.104 & 0.333 \\
\hline Recommendation & -0.070 & 0.561 & 0.073 & 0.496 \\
\hline Ephemerality & -0.241 & $0.047^{*}$ & -0.070 & 0.514 \\
\hline
\end{tabular}

Table 1: Effects of anonymity, recommendation, and ephemerality on 12 types of social costs. For p-value, ${ }^{*}$ means $<0.05$; * means $<0.01 ;{ }^{* * *}$ means $<0.001$.
Independent Variable. Anonymity, Recommendation, and Ephemerality. We have three variables to indicate (1) Anonymity, whether the question is distributed with anonymity or with real identity, (2) Recommendation, whether the question is distributed to system-picked users or tagged users, and

(3) Ephemerality, whether the question was inaccessible in 1 day or 7 days.

Modeling. We utilized fixed effects linear regression models to represent relationships between three features (anonymity, recommendation, and ephemerality) and each type of social costs.

The fact that we considered these analyses simultaneously might introduce multiple comparison problem. Hence, we addressed this issue by performing a $p$-value adjustment using the BenjaminiHochberg approach, and set false discovery rate to 0.2 as recommended by McDonald [9].

\section{RESULTS}

The mean and standard deviation of each type of social cost are shown in Figure 2. The average mean is 2.05 in 5 point scale. The average standard deviation is 1.19. As we can see, users were most concerned about indebtedness and least concerned about dependence in our study.

Table 1 shows the results of twelve linear regression models of three independent variables and twelve dependent variables. The results show that anonymity, recommendation, and ephemerality in general had negative associations with the types of social costs that we established. We only show the main effects of these three features without interaction terms between them because none of these interaction terms have significant effects. Anonymity reduced the social costs associated with self-image by $8.5 \%$ and with privacy by $2.6 \%$ but not significant for both $(\mathrm{p}=0.062$ and $\mathrm{p}=0.622$ ). However, anonymity had significant effects $(\mathrm{p}=0.022)$ on reducing the indebtedness by $9.0 \%$. We see that recommendation reduced social costs associated with effort by $8.1 \%$, but the effect was not significant $(p=0.101)$. In contrast, recommendation significantly $(p=0.016)$ reduced the aspect of consideration in social costs by $12.5 \%$. Ephemerality significantly $(\mathrm{p}=0.006)$ reduced social costs associated with self-image by $12.7 \%$, and also significantly $(\mathrm{p}=0.004)$ reduced social costs of privacy by $15.2 \%$.

\section{DISCUSSION AND FUTURE DEVELOPMENT}

Through a randomized controlled experiment, we examined the effects of anonymity, recommendation and ephemerality on different perspectives of social costs. We found that anonymity reduces social costs on indebtedness, recommendation reduces social costs associated with consideration, and ephemerality reduces social costs regarding privacy and self-image concerns. Our study shows the perceived social costs play an important role in affecting user activity level in online settings such as QA sites, and demonstrates the importance of incorporating social costs in future online community building as a design factor. 
We also acknowledge several limitations in the current study that will guide our future development. First, we observe that study participants tend to ask generic "low-cost" questions (e.g., not sensitive or personal, see examples in Figure 1), which might explain why some of the results are not significant. In the future development, instead of randomly assigning participants to one of the eight conditions, we will allow participants to choose how they'd like to post the question by turning on or off the three features for a more natural setup. Second, we will continue the snow sampling to recruit more participants of diverse backgrounds to construct a social network at scale for an experiment of a longer timespan.

\section{REFERENCES}

[1] Stella E Anderson and Larry J Williams. 1996. Interpersonal, job, and individual factors related to helping processes at work. Journal of Applied Psychology 81, 3 (1996), 282.

[2] Lisa J Barney, Kathleen M Griffiths, Anthony F Jorm, and Helen Christensen. 2006. Stigma about depression and its impact on help-seeking intentions. Australian and New Zealand Journal of Psychiatry 40, 1 (2006), 51-54.

[3] Joseph B Bayer, Nicole B Ellison, Sarita Y Schoenebeck, and Emily B Falk. 2016. Sharing the small moments: ephemeral social interaction on Snapchat. Information, Communication \& Society 19, 7 (2016), 956-977.

[4] Erin L Brady, Yu Zhong, Meredith Ringel Morris, and Jeffrey P Bigham. 2013. Investigating the appropriateness of social network question asking as a resource for blind users. In Proceedings of the 2013 conference on Computer supported cooperative work. ACM, 1225-1236.

[5] Peter R Druian and Bella M DePaulo. 1977. Asking a child for help. Social Behavior and Personality: an international journal 5, 1 (1977), 33-39.

[6] Brian Frey and Carolyn Seaman. 2015. Scientists tell stories: About seeking help with programming. In Visual Languages and Human-Centric Computing (VL/HCC), 2015 IEEE Symposium on. IEEE, 47-51.

[7] Fiona Lee. 1997. When the going gets tough, do the tough ask for help? Help seeking and power motivation in organizations. Organizational behavior and human decision processes 72, 3 (1997), 336-363.

[8] Fiona Lee. 2002. The social costs of seeking help. The Journal of Applied Behavioral Science 38, 1 (2002), 17-35

[9] John H McDonald. 2009. Handbook of biological statistics. Vol. 2. Sparky House Publishing Baltimore, MD.

[10] Jeffrey M Rzeszotarski and Meredith Ringel Morris. 2014. Estimating the social costs of friendsourcing. In Proceedings of the 32nd annual ACM conference on Human factors in computing systems. ACM, 2735-2744.

[11] E Gary Shapiro. 1980. Is seeking help from a friend like seeking help from a stranger? Social Psychology Quarterly (1980), 259-263.

[12] Jiliang Tang, Xia Hu, and Huan Liu. 2013. Social recommendation: a review. Social Network Analysis and Mining 3, 4 (2013), 1113-1133.

[13] Edwina S Uehara. 1995. Reciprocity reconsidered: Gouldner'smoral norm of reciprocity'and social support. Journal of Social and Personal Relationships 12, 4 (1995), 483-502.

[14] David L Vogel and Stephen R Wester. 2003. To seek help or not to seek help: The risks of self-disclosure. Journal of Counseling Psychology 50, 3 (2003), 351.

[15] Svetlana Yarosh. 2013. Shifting dynamics or breaking sacred traditions?: the role of technology in twelve-step fellowships. In Proceedings of the SIGCHI Conference on Human Factors in Computing Systems. ACM, 3413-3422. 